\title{
SCIDOC
}

\author{
International Journal of Dentistry and Oral Science (IJDOS) \\ ISSN: $2377-8075$
}

\section{Comparative Evaluation of Tensile and Compressive Strength Of Cention N And Resin Modified Glass Ionomer Cements}

Research Article

Jose Jacob ${ }^{1}$, Malvika Sisodia ${ }^{2}$, Swarup Shetty ${ }^{3}$, Prashant Viragi ${ }^{4}$, Azhar Mohammed ${ }^{5 *}$, Shaik Riyaz Basha ${ }^{6}$

${ }^{1}$ Associate Professor, Department of Conservative Dentistry \& Endodontics, Pushpagiri College of Dental Sciences, Perumthuruthy PO, Thiruvalla, Kerala, India.

${ }^{2}$ Associate Professor, Department of Conservative Dentistry and Endodontics, Mithila Minority Dental College \& Hospital, Darbanga, India.

${ }^{3}$ Assistant Professor, Department of Prosthodontics and Crown \& Bridge, Sharavathi Dental College and Hospital, Alkola, Shimoga, Karnataka, India.

${ }^{4}$ Professor, Department of Public Health Dentistry, Rural Dental College, PIMS, Loni, Maharashtra, India.

${ }^{5}$ Lecturer, Department of Orthodontics and Dentofacial Orthopedics, Nitte (Deemed to be University), AB Shetty Memorial Institute of Dental Sciences (ABSIMDS), Mangalore, Karnataka, India.

${ }^{6}$ Department of Prosthodontics, Mallareddy Institute of Dental Sciences, Hedarabad, Telangana, India.

\section{Abstract}

Introduction: Restoration of the tooth structure is essential for the proper functioning of the teeth and prevention of further loss of tooth structure. The ideal requirement for restorative material should be good compressive and tensile strength. Materials and Methods: A total of 40 specimens were fabricated with 20 samples of RMGIC (GC FujiCEM®2) and Cention N (Cention Ivoclar Vivadent, Mumbai, India) to test the compressive strength and flexural strength. Restorative material samples were fabricated using cylindrical aluminum and later it was subjected to compressive and tensile strength using universal testing machine.

Results: Cention N had statistically significant higher mean compressive strength (118.97 MPa) compared to RMGIC (60.90 $\mathrm{MPa}$ ). Cention $\mathrm{N}$ had significantly higher tensile strength (12.32 \pm 3.02$)$ compared to RMGIC (7.72 \pm 2.64$)$.

Conclusion: Cention N had higher compressive and tensile strength compared to RMGIC, hence it can be advised as posterior restorative material in clinical practice.

Keywords: Cention N; Compressive Strength; RMGIC; Tensile Strength.

\section{Introduction}

Dental caries is a most common dental dieses affecting hard structure of a tooth resulting into destruction of tooth structure. Restoration of the carious tooth structure is necessary for the proper functioning of the teeth and to prevent of further loss of tooth structure. The ideal restorative material should have good compressive strength, diametral tensile strength, shear bond strength, adhesive, tooth colored, resistant to wear, nontoxic, biocompatible to the tissue and least microleakage for longevity of a restoration. $[1,2]$ Choice of restorative materials is made, based on, adhesion to the tooth structure, physical and mechanical properties, biocompatibility, and simplicity of application.[3] It is said that compressive strength is the most important mechanical property of restorative materials. A material with very low compressive strength than tooth, tends to fracture under occlusal loads. [2] Tensile and shear stresses generated in cervical region of the teeth causes cuspal flexure, interrupts the bonds amongst hydroxyapatite crystals of tooth, resulting in to formation of crack.4Hence restorative material should have good physical properties. Various restorative materials are available in the market such as; glass ionomer cement (GIC), amalgam, composites, cention $\mathrm{N}$, and resin modified GIC.

In 1972 by Wilson and Kent Glass introduced glass ionomer cement (GIC). GIC has unique properties such as; adhesion to tooth structure, biocompatible, and anticarcinogenic properties due fluoride. Conversely, one of the main disadvantages of GIC is its deprived mechanical charactersfor example, lower compres-

\section{*Corresponding Author:}

Dr. Azhar Mohammed,

Lecturer, Department of Orthodontics and Dentofacial Orthopedics, Nitte (Deemed to be University), AB Shetty Memorial Institute of Dental Sciences (ABSIMDS), Mangalore, Karnataka, India.

E-mail: azharmohammad@gmail.com

Received: May 09, 2021

Accepted: July 22, 2021

Published: August 14, 2021

Citation: Jose Jacob, Malvika Sisodia, Swarup Shetty, Prashant Viragi, Azhar Mohammed, Shaik Rivaz Basha. Comparative Evaluation of Tensile and Compressive Strength Of Cention N And Resin Modified Glass Ionomer Cements. Int J Dentistry Oral Sci. 2021;8(8):3670-3673. doi: http://dx.doi.org/10.19070/2377-8075-21000751

Copyright: Azhar Mohammed 2021 . This is an open-access article distributed under the terms of the Creative Commons Attribution License, which permits unrestricted use, distribution and reproduction in any medium, provided the original author and source are credited. 
sive strength and brittleness.[3] To overcome the inferior mechanical properties of associated with conventional glass ionomer cements, resin modification of glass-ionomer cement (RMGIC) was designed to achieve desirable physical properties. [5]

RMGIC is a hybrid restorative material which has fluoro-aluminosilicate glass particles, water-soluble methacrylate monomer with photoinitiator, polyacrylic acid, and water. RMGICs have ion-releasing filler glass particles.[4]

Cention N (Ivoclar Vivadent) is a newly developed tooth-colored, "alkasite" restorative material for bulk placement with or without the application of an adhesive. It is basically a subsection of the composite resin. Cention $\mathrm{N}$ is a urethane dimethacrylate (UDMA)-based, self-curing powder/liquid type restorative material with electiveadded light curing advantages. Cention $\mathrm{N}$ displays a high polymer network density and degree of polymerization over the complete depth of the restoration.[2-6]

The purpose of this study was to evaluate the compressive and tensile strength of Cention $\mathrm{N}$ over resin modified GIC.

\section{Materials and Methods}

Present in vitro study was carried out in the department of Conservative dentistry. A total of 40 specimens were fabricated with 20 samples of RMGIC (GC FujiCEM ${ }^{\circledR}$ ) and Cention N (Cention Ivoclar Vivadent, Mumbai, India) to test the compressive strength and flexural strength.

\section{Compressive strength evaluation}

Cylindrical aluminum split molds of dimension $5 \pm 1 \mathrm{~mm}$ (height) $\times 4 \pm 1 \mathrm{~mm}$ (diameter) were used to fabricate 20 samples of each restorative material for testing the compressive strength. The samples were stored in a water bath for $24 \mathrm{hrs}$ at $37 \pm 1^{\circ} \mathrm{C}$ before testing. The samples were tested using universal testing machine at crosshead speed of $1.0 \mathrm{~mm} /$ minute.

\section{Diametral tensile strength (DTS) testing}

For fabrication of samples to evaluate tensile strength, cylindrical aluminum split molds of dimension $20 \pm 1 \mathrm{~mm}$ (length) $\times 2 \pm 1$ $\mathrm{mm}$ (height) $\times 2 \pm 1 \mathrm{~mm}$ (width) were chosen. The sample was subjected to tensile strength evaluation using universal testing machine. Maximum load was applied to the fracture, the specimens were noted at a crosshead speed of $0.1 \mathrm{~mm} /$ minute and the DTS was calculated using the following formula: $T=2 \mathrm{P} / \pi \mathrm{DL}$, where $\mathrm{P}$ is the maximum applied load $(\mathrm{N}), \mathrm{D}$ is the measured diameter of the sample $(\mathrm{mm})$, and $\mathrm{L}$ is the measured length of the sample (mm).

\section{Statistical Analysis}

The Null Hypothesis was considered, that there was no significant difference in the mean compressive and tensile strength between the two materials. Independent $t$ test was applied with alternate hypothesis of significant difference in the mean strength.

The values were tabulated and statistically analyzed using IBM SPSS statistical software version 22.0 Chicago, with one-way ANOVA and Tukey's post hoc test withp $<0.05$.

\section{Results}

Table 1 and 2 indicated that, cention $\mathrm{N}$ had statistically higher mean compressive strength (118.97 MPa) compared to RMGIC (60.90 MPa). Table 3 indicated the mean tensile bond strength among the cention and RMGCI restorative materials. Cention N had significantly higher tensile strength $(12.32 \pm 3.02)$ compared to RMGIC (7.72 \pm 2.64$)$.

Table 1. Mean compressive strength of two materials.

\begin{tabular}{|l|c|c|c|c|c|c|}
\hline \multirow{2}{*}{ Group } & \multirow{2}{*}{$\mathbf{N}$} & \multirow{2}{*}{ Mean } & \multirow{2}{*}{ SD } & \multirow{2}{*}{ SE } & \multicolumn{2}{|c|}{ 95\% confidence interval } \\
\cline { 6 - 7 } & & & & & Lower bound & Upper bound \\
\hline Cention & 20 & 118.97 & 0.971 & 7.423 & 102.74 & 134.86 \\
\hline RMGIC & 20 & 60.9 & 0.904 & 1.643 & 57.87 & 59.89 \\
\hline
\end{tabular}

SD- standard deviation, SE- standard error

Table 2. Difference in mean compressive strength between two materials.

\begin{tabular}{|l|c|c|c|c|c|c|}
\hline Groups & $\mathbf{N}$ & Range & Mean \pm SD & SEM & t value & P value \\
\hline Cention & 20 & $94.21-143.76$ & $118.97 \pm 24.21$ & 7.423 & 9.456 & $<0.001^{* *}$ \\
\cline { 1 - 5 } RMGIC & 20 & $56.32-65.47$ & $60.90 \pm 4.58$ & 1.643 & & \\
\hline
\end{tabular}

$\mathrm{P}<0.001, * *_{-}$highly significant

Table 3. Comparison of tensile strength.

\begin{tabular}{|c|c|c|c|}
\hline Material used & Number & Micro tensile bond strength & P value \\
\hline Cention & 20 & $12.32 \pm 3.02$ & \multirow{2}{*}{$<0.001$} \\
\hline RMGIC & 20 & $7.72 \pm 2.64$ & \\
\hline
\end{tabular}




\section{Discussion}

Several direct restorative materials are available to the modern dental practice from amalgams to modern bulk fill composites. A restorative material should provide sufficient tensile and compressive strength to resist multidirectional masticatory forces for many years. [6] The main purpose of the restorative materials is to substitute of the esthetic, biological, functional, and properties of healthy tooth structure is the.[7] Nowadays the usage of resinbased composite restoration has raised because of its excellent esthetic and other favorable characteristics.[3]

Venugopal et al assessed the microleakage in Class V cavity restored with resin modified GIC, nanohybrid flowable composite, and Cention N. They concluded that RMGIC had less microleakage in contrast to other tested materials. [4]

Kaur et al estimated the compressive strength of Cention $\mathrm{N}$ with glass ionomer cement. They concluded that cention $\mathrm{N}$ had higher strength than type IX GIC hence Cention $\mathrm{N}$ can be used as a superior alternative to GIC Type IX for posterior restoration.[2]

Iftikhar et al associated the mechanical properties (compressive strength (CS) and diametral tensile strength (DTS)) of four different restorative materials: ClearFil AP-X, conventional glass ionomer (Fuji IX), Filtex Z350-XT, and Cention N.They concluded that, ClearFil AP-X has the highest mechanical properties.[3]

Mishra et al related the compressive and flexural strength of Centurion $\mathrm{N}$ with GIC, and amalgam. They concluded that compressive strength of cention $\mathrm{N}$ was expressively higher than GIC. Flexural strength of cention $\mathrm{N}$ was observed to be suggestively greater than GIC and Amalgam.[6]

Feiz et al associated the micro tensile bond strength of four glasscontaining materials on primary teeth.There was no substantialvariance in the outline of the failure among groups, except RMGI and cention N. In contrast to our results they found equal strength between cention $\mathrm{N}$ and RMGI.[7]

Rekha et al. associated tensile bond strength and microleakage of conventional glass ionomer cement, RMGI and compomer to primary tooth and found highest tensile bond strength with compomer and the least tensile bond strength for RMGI.[8]

Naz1 et al. evaluated the compressive strength and microleakage of GIC type IX, Zirconomer Improved and Cention N. Cention $\mathrm{N}$ had the least microleakage followed by Zirconomer improved and GIC type IX. These results are similar to our findings.[1]

Eligeti et al assessed the shear bond strength of Cention $\mathrm{N}$ and recent tooth-colored restorative materials to dentin. Cention $\mathrm{N}$ exhibited highest mean Shear Bond strength compared to KetacMolar, and Zirconomer Improved. They determined that Cention $\mathrm{N}$ restorative material had better bonding capacity in comparison to Zirconomer Improved, RMGIC, and Ketac-Molar.[5]

Gurveen Kaur et al evaluated the microleakage and compressive strength of conventional GIC, modifications of GIC with chitosan and chlorhexidine and Cention-N. Highest compressive strength and the lowest microleakage was observed in Cention. [9]
Moshaverinia et al assessed the diametral tensile, compressive, and flexural strengths of a reinforced glass ionomer dental restorative material (EQUIA Forte Fil with Fuji IX GP and ChemFil Rock). They concluded that EQUIA Forte Fil had superior flexural strength and surface hardness. [10]

Arun Kumar and Ajitha compared the compressive strength of Cention $\mathrm{N}$ and high copper amalgam. There was no statistically substantial variance between Cention- $\mathrm{N}$ and amalgam for compressive strength.[11]

Kim et al estimated the physical properties and shear bond strength of two resin-modified glass-ionomers: RMGIs (FJL and VT) and as a control, a conventional glass-ionomer: CGI (FJ), and found no significant alteration between the tested groups.[12] Forouzanmehr et al measured the effect of adding purified powder of Salvia officinalis on the mechanical properties and bonding ability of resin-modified glass ionomer (RMGI) cement and concluded that, there is no difference with addition of purified powder of S. officinalis to RMGI powder.[13] Verma et al associated the shear bond, compressive strength, and microhardness of GIC and Cention $\mathrm{N}$ and determined that shear bond strength of Cention $\mathrm{N}$ was statistically highly significant as compared to GIC Type IX.[14]

Balagopal et al evaluated the shear bond strength, flexural strength, and fluoride-releasing capacity of GIC,with Cention N. They determined that Flexural strength of Cention $\mathrm{N}^{\circledR}$ was expressively greater in comparison to Fuji IX GIC ${ }^{\circledR}$ and there were no substantial alterations in shear bond strength of both the materials. [15] In the present study we found improved compressive and tensile strength with cention-N compared to RMGIC. Compressive strength of Cention $\mathrm{N}$ was found to be highest because of presence of UDMA particles in the monomer matrix which is less elastic and provides stiffness to the matrix. Cention $\mathrm{N}$ had the minimum microleakage amongst several group. The reason may be because, Cention $\mathrm{N}$ has a special patented filler isofiller, which is partially functionalized by silanes.1Cention $\mathrm{N}$ has hydroperoxide and the filler in the powder is coated with the other initiator components. Instead of conventional benzoyl peroxide, Hydroperoxide imparts greater temperature-resistance i.e. it is less sensitive to heat, which is an important factor regarding storage stability.[5]

The drawback of the present study was; it compared only two materials and it was in vitro study. Further in vivo studies are required to evaluate the mechanical properties of the various restorative materials.

\section{Conclusion}

Cention $\mathrm{N}$ had higher compressive and tensile strength compared to RMGIC, hence it can be advised as posterior restorative material in clinical practice.

\section{References}

[1]. Naz T, SingDJ, Somani R, Jaidka S.Comparative evaluation of microleakage and compressive strength of glass ionomer cement type ix, zirconomer improved and cention N- an in vitro study.Int. J. Adv. Res.2019; 7(9): 921-931

[2]. Kaur M, Mann NS, Jhamb A, Batra D. A comparative evaluation of compressive strength of Cention $\mathrm{N}$ with glass Ionomer cement: An in-vitro 
study. Int J Appl Dent Sci. 2019;5(1):05-9.

[3]. Iftikhar N, Devashish, Srivastava B, Gupta N, Ghambir N, Rashi-Singh. A Comparative Evaluation of Mechanical Properties of Four Different Restorative Materials: An In Vitro Study. Int J Clin Pediatr Dent. 2019 JanFeb;12(1):47-49.Pubmed PMID: 31496572.

[4]. Venugopal K, Krishnaprasad L, Singh VP, Ravi AB, Haridas K, Soman D. A comparative evaluation of microleakage between resin-modified glass ionomer, flowable composite, and cention- $\mathrm{N}$ in Class $\mathrm{V}$ restorations: A confocal laser scanning microscope study. J Pharm Bioall Sci. 2021 Jun 1;13(5):1326.

[5]. Eligeti T, Dola B, Kamishetty S, Gaddala N, Swetha A, Bandari J. Comparative Evaluation of Shear Bond Strength of Cention N with Other Aesthetic Restorative Materials to Dentin: An in Vitro Study. Annals of R.S.C.B. 2021 Jun 20;25(6):12707-14.

[6]. Mishra A, Singh G, Singh S, Agarwal M, Qureshi R, Khurana N. Comparative evaluation of mechanical properties of Cention $\mathrm{N}$ with conventionally used restorative materials-an in vitro study. Int J Prosthodont Restor Dent. 2018;8(4):120-4.

[7]. Feiz A, Amrollahi N, Ziayi F. Comparative evaluation of microtensile bond strength of four glass-containing materials with primary teeth dentin. Iran J Pediatr. 2019 Aug 31;29(4): e88774.

[8]. Rekha CV, Varma B, Jayanthi. Comparative evaluation of tensile bond strength and microleakage of conventional glass ionomer cement, resin modified glass ionomer cement and compomer: An in vitro study. Contemp Clin Dent. 2012 Jul;3(3):282-7.Pubmed PMID: 23293482.
[9]. Kaur G, Nankani HS, Mavishna MV, Venkatesh KV. A comparative evaluation of microleakage and compressive strength of Glass Ionomer Cement, modifications of Glass Ionomer Cements and Cention N- An Invitro study. IJDSIR. 2020; 3(3): 457 - 467.

[10]. Moshaverinia M, Navas A, Jahedmanesh N, Shah KC, Moshaverinia A, Ansari S. Comparative evaluation of the physical properties of a reinforced glass ionomer dental restorative material. J Prosthet Dent. 2019 Aug 1;122(2):154-9.

[11]. Kumar SA, Ajitha P. Evaluation of compressive strength between Cention N and high copper amalgam-An in vitro study. Drug invent. today. $2019 \mathrm{Feb}$ 15;12(2):255-257.

[12]. KIM YG, HIRANO S, HIRASAWA T. Physical properties of resin-modified glass-ionomers. Dent Mater J. 1998;17(1):68-76.

[13]. Forouzanmehr M, Barekatain M, Shahtalebi MA. An in vitro evaluation of the compressive strength and shear bond strength of resin-modified glassionomer cement containing purified powder of Salvia officinalis. Dent Res J (Isfahan). 2020 Sep 7;17(5):319-325.Pubmed PMID: 33343839.

[14]. Verma V, Mathur S, Sachdev V, Singh D. Evaluation of compressive strength, shear bond strength, and microhardness values of glass-ionomer cement Type IX and Cention N. J Conserv Dent. 2020 Nov-Dec;23(6):550-553. Pubmed PMID: 34083907.

[15]. Balagopal S, Nekkanti S, Kaur K. An In Vitro Evaluation of the Mechanical Properties and Fluoride-releasing Ability of a New Self-cure Filling Material. J Contemp Dent Pract. 2021 Feb 1;22(2):134-139.Pubmed PMID: 34257171 . 